\title{
P03-6-17 Poster session
}

\section{The importance of peroxisome in obesity-related adipocyte injury}

\author{
Songling Jiang, Lingjuan Piao, Hunjoo Ha
}

\section{Graduate School of Pharmaceutical Sciences, College of Pharmacy, Ewha Womans University, Korea}

Background: Increased oxidative stress in adipose tissue is critical for the development of insulin resistance and metabolic syndrome in obesity. Peroxisomes are involved in fatty acid oxidation and removal of reactive oxygen species, but the importance of peroxisome homeostasis in adipose tissue is poorly understood. This study aimed to determine the importance of adipose peroxisomal biogenesis in obesity.

Methods: 8-week-old C57BL/6J mice and PPAR $\alpha^{\%}$ mice were fed with normal diet (ND) or HFD for 12 weeks, and FF $(50 \mathrm{mg} / \mathrm{kg} /$ day) was orally administered along with HFD initiation. Differentiated 3T3-L1 adipocytes were used to dissect the molecular mechanisms involved in peroxisomal homeostasis.

Results: Peroxisome-related gene expressions were significantly down-regulated in white adipose tissue (WAT) of diabetic obese $\mathrm{db} / \mathrm{db}$ mice, suggesting a close correlation between peroxisomes and the development of obesity. Furthermore, treatment with $\mathrm{H}_{2} \mathrm{O}_{2}$ or proinflammatory factor tumor necrosis factor alpha (TNF $\alpha$ ) was found to downregulate peroxisomal genes in adipocytes. Furthermore, direct down-regulation of pex 5 induced the increment of ROS production and resulted in the induction of inflammatory cytokines. In contrast, treatment with fenofibrate, a peroxisomal proliferator-activated receptor alpha (PPAR $\alpha)$ agonist, led to increased peroxisomal biogenesis in WAT and attenuated HFD-associated phenotype in WT mice but not in PPAR $\alpha$-deficient mice.

Conclusion: These data suggest that adipose peroxisomal homeostasis plays an important role in metabolic phenotype of obesity and that peroxisome may become a potential target for obesity treatment. 